\title{
PARAmeter Estimation of the Hybrid Censored Log-Normal Distribution
}

\author{
Sulabh Dube* Biswabrata Pradhan ${ }^{\dagger} \&$ Debasis Kundu $\ddagger$
}

\begin{abstract}
The two most common censoring schemes used in life testing experiments are Type-I and Type-II censoring schemes. Hybrid censoring scheme is mixture of Type-I and Type-II censoring scheme. In this work we consider the estimation of parameters of log-normal distribution based on hybrid censored data. The parameters are estimated by the maximum likelihood method. It is observed that the maximum likelihood estimates can not be obtained in closed form. We obtain the maximum likelihood estimates of the unknown parameters using EM algorithm. We also propose approximate maximum likelihood estimates and these can be used as initial estimates for any iterative procedure. The Fisher information matrix has been obtained and it can be used for constructing asymptotic confidence intervals. The method of obtaining optimum censoring scheme is discussed. One data set is analyzed for illustrative purposes.
\end{abstract}

Keywords: Maximum likelihood estimators, EM algorithm, Type-I censoring, Type-II censoring, optimal censoring scheme, approximate maximum likelihood estimate.

${ }^{*}$ Wipro Technologies, Sarjapur Road, Bangalore, Pin 560035, India.

${ }^{\dagger}$ SQC \& OR Unit, Indian Statistical Institute, 203 B.T. Road, Kolkata, Pin 700108, India. Corresponding author, e-mail: bis@isical.ac.in

${ }^{\ddagger}$ Department of Mathematics and Statistics, Indian Institute of Technology Kanpur, Pin 208016, India. 


\section{Introduction}

Type-I and Type-II censoring schemes are the two most popular censoring schemes which are used in practice. They can be briefly described as follows; Suppose $n$ units are put on a life test. In Type-I censoring, experiment continues up to a pre-specified time T. Failures after the time $T$ are not observed. In Type-II censoring, the experiment continues till the $R$-th failure takes place, where $R$ is a pre-specified integer and $R \leq n$. Therefore, in Type-I censoring scheme, the number of failures is random and in Type-II censoring scheme, the experimental time is random.

A mixture of Type-I and Type-II schemes is known as the hybrid censoring scheme. Suppose $n$ identical units are put on a life test. The test is terminated when a pre-specified number $R$, out of $n$ units have failed or a pre-determined time $T$, has been reached. Therefore, in hybrid censoring scheme, the experimental time and the number of failures will not exceed $T$ and $R$ respectively. It is clear that Type-I and Type-II censoring schemes can be obtained as special cases of hybrid censoring scheme by taking $R=n$ and $T=\infty$ respectively. It has been used quite effectively in reliability acceptance sampling test in MIL-STD-781C [23].

Epstein [10] first introduced the hybrid censoring scheme and analyzed the data under the assumption of exponential lifetime distribution of the experimental units. He also proposed a two sided confidence interval of the unknown parameter, without any formal proof. Fairbanks et al. [11] modified slightly the proposition of Epstein [10] and suggested a simple set of confidence intervals. Chen and Bhattacharya [4] obtained the exact one sided confidence interval based on the distribution of the maximum likelihood estimator of the exponential parameter. Drapper and Guttmann [7] also considered the same problem but from the Bayesian point of view, and obtained two sided credible interval of the mean lifetime using

the inverted gamma prior. Comparisons and criticisms of the different methods can be found 
in Gupta and Kundu [12]. For some of the relevant references on hybrid censoring and related topics the readers are referred to Ebrahimi [8, 9], Jeong et al. [14], Childs et al. [5], Kundu [16], Banerjee and Kundu [3], Kundu and Pradhan [18] and the references cited there.

It may be mentioned that although hybrid censoring scheme seems to be an important censoring scheme, but not much work has been done other than the exponential or Weibull distribution. In this paper we consider the hybrid censored lifetime data, when the lifetime follows two parameter log-normal distribution. The log-normal distribution has been used quite effectively in analyzing positively skewed data. Almost a book length treatment on log-normal distribution can be obtained from Johnson et al. [15].

We first try to obtain the maximum likelihood estimators (MLEs) of the unknown parameters. It is observed that the maximum likelihood estimates can be obtained by solving two non-linear equations, but they can not be obtained in closed form. Although, the standard Newton-Raphson algorithm can be employed to solve the non-linear equations, but unfortunately it does not converge all the time even from good starting values. Instead, we suggest to use the EM algorithm to compute the MLEs. Finding the initial estimates is an important problem in any iterative procedure. We have proposed approximate MLEs, which have explicit expressions in terms of the observations and they can be used as initial guesses for any iterative procedure. Simulation study is carried out to compare the performance of the MLEs and approximate MLEs (AMLEs). A data set has been analyzed for illustrative purpose. Finding the optimum censoring scheme is an important problem. We discuss how to compare two different hybrid censoring schemes in terms of their information measures and hence find the optimum censoring scheme.

The rest of the paper is arranged as follows. The model and data are described in Section 2. The maximum likelihood estimators and approximate maximum likelihood estimators of the unknown parameters are provided in Sections 3 and 4 respectively. Simulation results 
are presented in Section 5. A data set is analyzed in Section 6. We discuss optimal censoring schemes in Section 7. Finally conclusions appear in section 8.

\section{Model Description}

It is assumed that the lifetime random variable $Y$ has a log-normal distribution with the following probability density function (PDF)

$$
f(y ; \mu, \sigma)=\frac{1}{\sqrt{2 \pi} \sigma y} e^{-\frac{(\ln y-\mu)^{2}}{2 \sigma^{2}}} ; y>0 ;-\infty<\mu<\infty, \sigma>0
$$

If $Y$ has the log-normal distribution (1), $X=\ln Y$ has the normal distribution with the PDF

$$
f(x ; \mu, \sigma)=\frac{1}{\sqrt{2 \pi} \sigma} e^{-\frac{(x-\mu)^{2}}{2 \sigma^{2}}} ; \quad-\infty<x<\infty .
$$

Model (1) and Model (2) are equivalent models in the sense that the procedure developed under one model can be used for other model. Now we briefly describe the available data in a hybrid censoring scheme.

Suppose $n$ identical units are put on life test. Then under hybrid censoring scheme, we have one of the following two types of observations;

Case I: $\left\{y_{1: n}<\ldots<y_{R: n}\right\} \quad$ if $y_{R: n}<T$.

Case II: $\left\{y_{1: n}<\ldots<y_{d: n}\right\} \quad$ if $d<R$ and $y_{d: n}<T<y_{d+1: n}$ where $y_{1: n}<y_{2: n} \ldots$ denote the observed ordered failure times of the experimental units.

\section{MAXIMUM LIKELIHOOD ESTIMATORS}

In this section we discuss how to compute the MLEs of the model parameters $\mu$ and $\sigma$ based on the observation given in Section 2. For clarity first we write down the likelihood function 
in Case I and Case II separately. The likelihood function in Case I is given by

$$
l(\mu, \sigma) \propto\left(\frac{1}{\sigma}\right)^{R} \prod_{i=1}^{R} \exp \left\{-\frac{\left(\ln y_{i: n}-\mu\right)^{2}}{2 \sigma^{2}}\right\}\left[1-\Phi\left(\frac{\ln y_{R: n}-\mu}{\sigma}\right)\right]^{n-R},
$$

and for Case II, it is given by

$$
l(\mu, \sigma) \propto\left(\frac{1}{\sigma}\right)^{d} \prod_{i=1}^{d} \exp \left\{-\frac{\left(\ln y_{i: n}-\mu\right)^{2}}{2 \sigma^{2}}\right\}\left[1-\Phi\left(\frac{\ln T-\mu}{\sigma}\right)\right]^{n-d} .
$$

Here $0 \leq d \leq R$. Note that (3) and (4) can be combined and they can be written as follows;

$$
l(\mu, \sigma) \propto\left(\frac{1}{\sigma}\right)^{r} \prod_{i=1}^{r} \exp \left\{-\frac{\left(\ln y_{i: n}-\mu\right)^{2}}{2 \sigma^{2}}\right\}\left[1-\Phi\left(\frac{c-\mu}{\sigma}\right)\right]^{n-r},
$$

here

$$
r=\left\{\begin{array}{ccc}
R & \text { for } & \text { Case I } \\
d & \text { for } & \text { Case II }
\end{array} \quad \text { and } \quad c=\left\{\begin{array}{ccc}
\ln y_{R: n} & \text { for } & \text { Case I } \\
\ln T & \text { for } & \text { Case II }
\end{array}\right.\right.
$$

It may be noted that if $d=0$, the MLE does not exist. So from now on it is assumed that $d>0$. Taking the logarithm of (5), we obtain

$$
L(\mu, \sigma)=-r \ln \sigma-\frac{1}{2 \sigma^{2}} \sum_{i=1}^{r}\left(x_{i: n}-\mu\right)^{2}+(n-r) \ln \left[1-\Phi\left(\frac{c-\mu}{\sigma}\right)\right],
$$

where $x_{i: n}=\ln y_{i: n}$. Taking derivatives with respect to $\mu$ and $\sigma$ of (7), we obtain

$$
\begin{aligned}
& \frac{\partial L}{\partial \mu}=\frac{1}{\sigma^{2}} \sum_{i=1}^{r}\left(x_{i: n}-\mu\right)+(n-R) \frac{\phi\left(z^{*}\right)}{\sigma \Phi\left(-z^{*}\right)}=0 \\
& \frac{\partial L}{\partial \sigma}=-\frac{r}{\sigma}+\frac{1}{\sigma^{3}} \sum_{i=1}^{r}\left(x_{i: n}-\mu\right)^{2}+(n-r) \frac{z^{*} \phi\left(z^{*}\right)}{\sigma \Phi\left(-z^{*}\right)}=0
\end{aligned}
$$

where $z^{*}=\frac{c-\mu}{\sigma}, \Phi(\cdot)$ and $\phi(\cdot)$ are the cumulative distribution function (CDF) and the probability density function (PDF) of a standard normal variate respectively. Define $z_{R}=\frac{\ln y_{R: n}-\mu}{\sigma}$ and $V=\frac{\ln T-\mu}{\sigma}$, then $z^{*}=z_{R}$ in case I and $z^{*}=V$ in case II. It is clear that the likelihood equations are implicit in nature in both the cases. We need some numerical techniques to solve the simultaneous equations. We use the standard Newton-Raphson (NR) method, but unfortunately it does not converge all the times. We suggest to use the EM algorithm to compute the MLEs and it is described below. 


\subsection{EM Algorithm}

The EM algorithm, originally proposed by Dempster et al. [6], is a very powerful tool in handling the incomplete data problem. We treat this problem as a missing value problem similarly as in $\mathrm{Ng}$ et al. [24]. Let $X=\left(x_{1: n}, \ldots, x_{r: n}\right)$ be the observed data and $U=$ $\left(u_{1}, \ldots, u_{n-r}\right)$ be the censored data. For a given $r, u_{1}, \ldots, u_{n-r}$ are not observable. The censored data vector $U$ can be thought of as missing data and $W=(X, U)$ forms the complete data. Let $L_{c}(w ; \mu, \sigma)$ be the log-likelihood corresponding to complete data. Then

$$
L_{c}(w ; \mu, \sigma)=-n \ln \sigma-\frac{1}{2 \sigma^{2}} \sum_{i=1}^{n}\left(w_{i}-\mu\right)^{2}=-n \ln \sigma-\frac{1}{2 \sigma^{2}} \sum_{i=1}^{r}\left(x_{i: n}-\mu\right)^{2}-\frac{1}{2 \sigma^{2}} \sum_{i=1}^{n-r}\left(u_{i}-\mu\right)^{2} .
$$

In $E$-step one needs to compute the pseudo log-likelihood function given by

$$
\begin{aligned}
L_{s}(\mu, \sigma)= & -n \ln \sigma-\frac{1}{2 \sigma^{2}} \sum_{i=1}^{r}\left(x_{i: n}-\mu\right)^{2}-\frac{1}{2 \sigma^{2}} \sum_{i=1}^{n-r} E\left(\left(U_{i}-\mu\right)^{2} \mid U_{i}>c\right) \\
= & -n \ln \sigma-\frac{1}{2 \sigma^{2}} \sum_{i=1}^{r}\left(x_{i: n}-\mu\right)^{2}-\frac{n-r}{2 \sigma^{2}} B\left(c ; \mu_{(k)}, \sigma_{(k)}\right) \\
& +\frac{n-r}{2 \sigma^{2}}\left[A\left(c ; \mu_{(k)}, \sigma_{(k)}\right)-\mu^{2}\right]
\end{aligned}
$$

where

$$
\begin{aligned}
A(c ; \mu, \sigma) & =E(U \mid U>c)=\sigma Q+\mu \\
B(c ; \mu, \sigma) & =E\left(U^{2} \mid U>c\right)=\sigma^{2}(1+\xi Q)+2 \sigma \mu Q+\mu^{2} \\
\xi & =\frac{c-\mu}{\sigma}, \quad Q=\frac{\phi(\xi)}{\Phi(-\xi)} .
\end{aligned}
$$

In M-step we maximize (11) with respect to $\mu$ and $\sigma$. Let $\left(\mu_{(k)}, \sigma_{(k)}\right)$ be the estimate of $(\mu, \sigma)$ at the $k$ th stage, then $\left(\mu_{(k+1)}, \sigma_{(k+1)}\right)$ can be obtained as

$$
\begin{aligned}
& \hat{\mu}_{(k+1)}=\frac{1}{n}\left[\sum_{i=1}^{r} x_{i: n}+(n-r) A\left(c ; \mu_{(k)}, \sigma_{(k)}\right)\right] \\
& \hat{\sigma}_{(k+1)}=\left\{\frac{1}{n}\left[\sum_{i=1}^{r} x_{i: n}^{2}+(n-r) B\left(c ; \mu_{(k)}, \sigma_{(k)}\right)\right]-\hat{\mu}_{(k+1)}^{2}\right\}^{1 / 2} .
\end{aligned}
$$


One of the advantages of using EM algorithm is that it gives a measure of information in the censored (missing) data in a natural way through the missing information principle. Louis [22] developed a procedure for extracting the observed information matrix when the EM algorithm is used. Note that

$$
\text { Observed Information }=\text { Complete information }- \text { Missing Information }
$$

In this case, if we denote $\theta=(\mu, \sigma)^{\prime}, X=$ the observed data, $W=$ the complete data, $I_{W}(\theta)=$ the complete information, $I_{X}(\theta)=$ the observed information, $I_{W \mid X}(\theta)=$ the missing information, then

$$
I_{X}(\theta)=I_{W}(\theta)-I_{W \mid X}(\theta)
$$

The complete information matrix for the normal distribution is given by

$$
I_{W}(\theta)=\frac{n}{\sigma^{2}}\left(\begin{array}{ll}
1 & 0 \\
0 & 2
\end{array}\right)
$$

Now

$$
I_{W \mid X}(\theta)=(n-r) I_{W \mid X}^{*}(\theta)
$$

where $I_{W \mid X}^{*}(\theta)=$ Information matrix of a single observation for a truncated normal distribution. It can be written as follows, see Ng. et al. [24].

$$
I_{W \mid X}^{*}(\theta)=\left(\begin{array}{cc}
\frac{1}{\sigma^{2}}\left[1+\xi Q-Q^{2}\right] & \frac{1}{\sigma^{2}}[Q+\xi Q(\xi-Q)] \\
\frac{1}{\sigma^{2}}[Q+\xi Q(\xi-Q)] & \frac{1}{\sigma^{2}}[2+\xi Q(1-\xi Q+\xi)]
\end{array}\right) .
$$

Thus the observed information matrix is give by

$$
I_{X}(\theta)=\frac{n}{\sigma^{2}}\left(\begin{array}{cc}
1 & 0 \\
0 & 2
\end{array}\right)-\frac{n-r}{\sigma^{2}}\left(\begin{array}{cc}
\frac{1}{\sigma^{2}}\left[1+\xi Q-Q^{2}\right] & \frac{1}{\sigma^{2}}[Q+\xi Q(\xi-Q)] \\
\frac{1}{\sigma^{2}}[Q+\xi Q(\xi-Q)] & \frac{1}{\sigma^{2}}[2+\xi Q(1-\xi Q+\xi)]
\end{array}\right)
$$

The asymptotic variance-covariance matrix of the maximum likelihood estimate of $\hat{\theta}$ can be obtained by inverting the observed information matrix $I_{X}(\hat{\theta})$. 


\section{Approximate MLE}

It is observed that the likelihood equations are not linear due to the presence of the term $h\left(z^{*}\right)=\frac{\phi\left(z^{*}\right)}{\Phi\left(-z^{*}\right)}$. Tiku et al. [26] discussed some approximate solutions in similar cases of non-linearity introduced due to some term/terms. Balakrishnan et al. [1] used the Taylor's series expansion of the term for progressively censored data from Gaussian distribution. Recently Kundu [16] and Banerjee and Kundu [3] also suggested similar approximations to compute the approximate MLEs of Weibull parameters when the data are hybrid censored. We first consider approximate MLEs for Case I. We expand the function $h\left(z_{R}\right)=\frac{\phi\left(z_{R}\right)}{\Phi\left(-z_{R}\right)}$ around $\nu=\Phi^{-1}\left(\frac{R}{n+1}\right)$ in Taylor series. Considering only the first order derivatives and neglecting the higher order derivatives we get

$$
h\left(z_{R}\right) \approx h(\nu)+\left(z_{R}-\nu\right) h^{\prime}(\nu)=\alpha+\beta z_{R}, \quad \text { (say) }
$$

where $\alpha=h(\nu)-\nu h^{\prime}(\nu)$ and $\beta=h^{\prime}(\nu) \geq 0$. So (8) can be written as

$$
\frac{\partial L}{\partial \mu} \approx \frac{1}{\sigma^{2}} \sum_{i=1}^{R}\left(x_{i: n}-\mu\right)+(n-R) \frac{\alpha+\beta z_{R}}{\sigma}=0 .
$$

This implies

$$
\begin{aligned}
\mu= & \frac{\sum_{i=1}^{R} x_{i: n}+(n-R) \beta x_{R: n}+(n-R) \alpha \sigma}{R+(n-R) \beta} \\
& =K+M \sigma, \text { say },
\end{aligned}
$$

where

$$
K=\frac{\sum_{i=1}^{R} x_{i: n}+(n-R) \beta x_{R: n}}{R+(n-R) \beta} \quad \text { and } \quad M=\frac{(n-R) \alpha}{R+(n-R) \beta} .
$$

From (9) we have

$$
\frac{\partial L}{\partial \sigma} \approx-\frac{R}{\sigma}+\frac{1}{\sigma^{3}} \sum_{i=1}^{R}\left(x_{i: n}-\mu\right)^{2}+(n-R) \frac{z_{R}}{\sigma}\left(\alpha+\beta z_{R}\right)=0
$$

This implies, on simplification,

$$
R \sigma^{2}-A_{1} \sigma-A_{2}=0,
$$


where

$$
A_{1}=(n-R) \alpha\left(x_{R: n}-K\right) \quad \text { and } A_{2}=\sum_{i=1}^{R}\left(x_{i: n}-K\right)^{2}+(n-R) \beta\left(x_{R: n}-K\right)^{2} \geq 0 .
$$

Clearly, (14) is a quadratic equation in $\sigma$. The solution for $\sigma$ is given by

$$
\hat{\sigma}=\frac{A_{1} \pm \sqrt{A_{1}^{2}+4 R A_{2}}}{2 R} .
$$

In this case one of the solutions will be positive and other one would be negative and we take the positive one. Once $\hat{\sigma}$ is obtained, estimate of $\mu$ as

$$
\hat{\mu}=K+M \hat{\sigma}
$$

The approximate MLEs can be used as initial estimates for the EM algorithm. We also study the performance of approximate MLEs through simulation study in the next section. The observed information matrix for the approximate MLE can be determined as follows.

$$
I_{2}=\left(\begin{array}{cc}
-\frac{\partial^{2} L}{\partial \mu^{2}} & -\frac{\partial^{2} L}{\partial \mu \partial \sigma} \\
-\frac{\partial^{2} L}{\partial \mu \partial \sigma} & -\frac{\partial^{2} L}{\partial \sigma^{2}}
\end{array}\right)
$$

where

$$
\begin{aligned}
\frac{\partial^{2} L}{\partial \mu^{2}} & \approx-\frac{R}{\sigma^{2}}-\frac{(n-R) \beta}{\sigma^{2}} \\
\frac{\partial^{2} L}{\partial \sigma^{2}} & \approx \frac{R}{\sigma^{2}}-\frac{3}{\sigma^{2}} \sum_{i=1}^{R}\left(\frac{x_{i: n}-\mu}{\sigma}\right)^{2}-(n-R) \frac{z_{R}}{\sigma^{2}}\left[2 \alpha+3 \beta z_{R}\right] \\
\frac{\partial^{2} L}{\partial \mu \partial \sigma} & \approx-\frac{2}{\sigma^{2}} \sum_{i=1}^{R}\left(\frac{x_{i: n}-\mu}{\sigma}\right)-\frac{(n-R)}{\sigma^{2}}\left[\alpha+2 \beta z_{R}\right]
\end{aligned}
$$

For Case II, we take the Taylor series expansion of $h(V)$ around $\nu=\Phi^{-1}\left(\frac{d+0.5}{n+1}\right)$. Other derivations in Case II is similar to Case I. In this case

$$
\begin{gathered}
K=\frac{\sum_{i=1}^{d} x_{i: n}+(n-d) \beta \ln T}{d+(n-d) \beta} \quad \text { and } \quad M=\frac{(n-d) \alpha}{d+(n-d) \beta}, \\
A_{1}=(n-d) \alpha(\ln T-K) \quad \text { and } \quad A_{2}=\sum_{i=1}^{d}\left(x_{i: n}-K\right)^{2}+(n-d) \beta(\ln T-K)^{2} \geq 0 .
\end{gathered}
$$

The observed information matrix can be easily obtained like as in Case I. 


\section{Simulation Study}

In this section we carry out a simulation study to compare the performances of the MLEs and the AMLEs. We take $\mu=4$ and $\sigma=0.15$ in all the ceases. The simulation is carried out for different choices of $n, R$ and $T$ values. For a particular set of hybrid censored data, the MLEs and AMLEs are obtained as described before. We replicate the process for 10000 times and report the average estimates, mean squared error (MSE) and 95\% coverage percentage of asymptotic confidence intervals. The results are reported in Tables 1 - 4. In each Table, the first and second row represent the result for $\mu$ and $\sigma$ respectively. From the simulation study it is observed that the AMLE performs well as per with the MLE. In fact they are very close to each other. As far as different choices of hybrid censoring schemes are concerned, (i) For fixed $n$ and $R$, when $T$ increases, the MSE decreases.

(ii) For fixed $n$ and $T$ as $R$ increases, no specific pattern observed in MSE. It can be due to the fact that as $R$ increases for fixed $T$, no additional information are gathered.

\section{Data Analysis}

In this section we analyze a data to illustrate our methodology. The data have been taken from Lawless [20] and shows the number of thousand miles at which different locomotive controls failed in a life test involving 96 controls. The test was terminated after 135,000 miles, by which time 37 failures had occurred. The failure times for the 37 failed units are: $22.5,37.5,46.0,48.5,51.5,53.0,54.5,57.5,66.5,68.0,69.5,76.5,77.0,78.5,80.0,81.5,82.0$, 83.0, 84.0, 91.5, 93.5, 102.5, 107.0, 108.5, 112.5, 113.5, 116.0, 117.0, 118.5, 119.0, 120.0, 122.5, 123.0, 127.5, 131.0, 132.5 and 134.0. In addition, there are 59 censoring times, all equal to 135.0 .

Clearly, this is a case of Type-I Censoring. In this case we have $n=96, T=135, d=37$ 
and $R>37$. The MLEs, AMLEs and the corresponding variance-covariance matrices are as follows;

$$
\begin{array}{cccc}
\text { MLEs: } & \hat{\mu}=5.117, \quad \hat{\sigma}=0.705, \quad V_{1}=\left(\begin{array}{cc}
0.01085 & 0.00573 \\
0.00573 & 0.00870
\end{array}\right) . \\
\text { AMLEs: } & \hat{\mu}=5.117, \quad \hat{\sigma}=0.705, \quad V_{2}=\left(\begin{array}{ll}
0.01082 & 0.00572 \\
0.00572 & 0.00870
\end{array}\right) .
\end{array}
$$

It is interesting to observe that the MLEs and AMLEs are same although there is a slight difference in the variance covariance matrices. One question arises whether the data fits log-normal distribution or not. To check for goodness of fit first we compute the KolmogorvSmirnov distance between the empirical distribution function and the fitted distribution function. It is 0.138 and the associated $p$ value is 0.481 . Since the $p$ value is quite high, we cannot reject the null hypothesis that the data are coming from the log-normal distribution. We further we provide PP plot in Figure 1. In PP plot, the X-axis represents the KaplanMeier estimate of the distribution function and $\mathrm{Y}$-axis represents the fitted distribution function based on the estimated parameters. The Figure also shows that the data fits reasonably well.

For illustrative purposes, we analyze another generated data set. We have generated the following observations, from the above data set based on the hybrid censoring scheme $(n=96, T=135, R=30) ; 22.5,37.5,46.0,48.5,51.5,53.0,54.5,57.5,66.5,68.0,69.5$, $76.5,77.0,78.5,80.0,81.5,82.0,83.0,84.0,91.5,93.5,102.5,107.0,108.5,112.5,113.5$, 116.0, 117.0, 118.5, 119.0. The estimated parameters and the variance-covariance matrices are given below.

$$
\begin{array}{ccc}
\text { MLEs: } & \hat{\mu}=5.303, \hat{\sigma}=0.847 . \quad V_{1}=\left(\begin{array}{cc}
0.02133 & 0.01293 \\
0.01293 & 0.01640
\end{array}\right) \\
\text { AMLEs: } & \hat{\mu}=5.303, \hat{\sigma}=0.847 . \quad V_{2}=\left(\begin{array}{ll}
0.02149 & 0.01298 \\
0.01298 & 0.01641
\end{array}\right) .
\end{array}
$$


As expected, we can see higher values in variance-covariance matrices when compared to Type-I Censoring. This is attributed to the fact that we had used fewer number of observations. In this case also, interestingly the MLEs and AMLEs are exactly the same, however, there is a slight difference in variance-covariance matrices as before.

\section{Optimal Censoring Scheme}

In the previous sections we have discussed statistical inferences of the unknown parameters for a given hybrid censoring scheme. In practice it is quite important to choose the optimum censoring scheme among all possible hybrid censoring schemes. Recently, finding the optimum censoring scheme has received some attention in the statistical literature. Some of the references for choosing optimal censoring schemes in different problems can be found in Lam [19], Lin et al. [21], Ng et al. [25], Zhang and Meeker [27], Balasooriya and Balakrishnan [2] and Kundu [17].

Note that the class of all hybrid censoring schemes can be expressed as;

$$
\mathcal{H}=\{(n, R, T) ; n \geq 1,1 \leq R \leq n, T>0\} .
$$

To find the optimum censoring scheme first we need to compare two different hybrid censoring schemes. Between two hybrid censoring schemes, it is natural to choose that particular hybrid censoring scheme which provides more information of the unknown parameters. Thus it is important to define the information measure for a given censoring scheme. A natural choice in such a case would be to compare the two Fisher Information Matrices. This is a very trivial task in case of a two parameter distribution if one parameter is already known. Because in such a case the whole exercise simply leads to the comparison of two real numbers. However, in most real life problems, both the parameters are unknown. In such cases, comparison of the two Fisher Information Matrices is not a trivial issue. One solution in this case could be 
to compare the traces or determinants of the two Fisher information matrices. This solution, though very simple, has an associated disadvantage that it is not scale invariant, see Gupta and Kundu [13]. Alternatively, Zhang and Meeker [27] proposed to compare the precision of $100 p$-th quantile estimators. Following their approach, Gupta and Kundu [13], see Kundu [17] also, proposed a more general information measure

$$
I(n, R, T)=\left[\int_{0}^{1} V\left((n, R, T)_{p}\right) d W(p)\right]^{-1},
$$

where $V\left((n, R, T)_{p}\right)$ denotes the asymptotic variance of the $100 p$-th quantile point, obtained using the censoring scheme $(n, R, T)$ and $W(\cdot)$ is any non-negative weight function such that $\int_{0}^{1} d W(p)=1$. To obtain the variance expression $V\left((n, R, T)_{p}\right)$, we need to derive expected Fisher information matrix. The expected Fisher information matrix is derived in Appendix. It is clear that $I(n, R, T)$ is independent of $p$. The weight function $W(\cdot)$ depends on the practitioner, moreover Zhang and Meeker's information measure can be obtained as a special of (15), see Kundu [17] for more details about choosing the weight function $W(\cdot)$.

It is observed in the numerical study, although we could not prove it theoretically, that for fixed $n$ and $T$ as $R$ increases or for fixed $n$ and $R$ as $T$ increases the information measure (15) first increases and then it becomes constant. Moreover, if $n=R$, and as $n$ and $T$ increases, (15) increases. Therefore, among the different hybrid censoring sachems, if somebody wants to choose that particular scheme which provides the maximum (15), the obvious choice will be $R=n=T=\infty$. It seems, without some proper restrictions on $n, R$ and $T$, the problem is of no use. In most of the practical situations, the obvious restrictions are on time and on the number of items used. We use the cost function approach similar as in Lam [18] and Lin et al. [21]. Let $C_{1}$ and $C_{2}$ denote the cost per unit time and per unit item respectively. Ignoring other associated costs, the total cost involved for a given hybrid censoring scheme becomes

$$
C_{0}=C_{1} T+C_{2} n
$$


To choose the optimum censoring scheme, the experimenter needs to know the unknown parameters. In practice, if it is completely unknown, the experimenter needs to perform some pilot study to approximate the unknown parameters. Therefore the problem boils down as follows: for a given $C_{0}$, among the class of all possible $(n, T)$, which satisfy (16) find $(n, R, T)$, which has the maximum (15). It is not possible to find the optimum value analytically, we need to find it numerically. Note that this optimization problem can be solved in finite number of steps as follows:

- Step 1: Find $n_{1}$, such that $n_{1}=\max \left\{n ; C_{2} n \leq C_{0}\right\}$.

- Step 2: Find the corresponding $T$ s, say $T_{1}, \ldots, T_{n_{1}}$, so that

$$
C_{1} T_{n}+C_{2} n=C_{0}, \quad \text { for } \quad n=1, \ldots, n_{1} .
$$

- Step 3: For each $\left(n, T_{n}\right)$ find $0<R_{n} \leq n$, so that the hybrid censoring scheme $\left(n, R_{n}, T_{n}\right)$ provides the maximum (15).

- Step 4: Among all possible choices of $\left(n, R_{n}, T_{n}\right)$, for $1 \leq n \leq n_{1}$, choose that particular scheme which provides the maximum (15).

For illustrative purpose, let us consider the following example. Suppose $\mu=0, \sigma=1$, $d W(p)=1$ for all $0 \leq p \leq 1$, and $C_{0}=100, C_{1}=10$ and $C_{2}=12$. In this case $n_{1}=8$. The information measure for different values of $n, R$ and $T$ are provided in the following Table 5. Therefore, in this case the optimum censoring plan is $(n, R, T)=(7,7,1.6)$.

\section{Conclusions}

In this paper we have considered the classical inference procedure for the unknown parameters of the log-normal distribution when the data are hybrid censored. It is observed that the 
maximum likelihood estimators can not be obtained in closed form and we have proposed to use the EM algorithm to compute the maximum likelihood estimators. We have proposed the approximate maximum likelihood estimators also, which can be obtained explicitly. We compare the performances of the different methods by Monte Carlo simulations and it is observed that the performances are quite satisfactory. We have also addressed the problem of finding the optimum censoring scheme. Extensive tables for different optimal censoring schemes are available with the authors and then can be obtained on request.

\section{ACKNOWLEDGEMENTS:}

The authors would like to thank the referee and the associate editor for constructive suggestions, which has improved the earlier draft of the manuscript. Part of the work of the third author has been supported by a grant from the Department of Science and Technology, Government of India.

\section{References}

[1] Balakrishnan, N., Kannan, N., Lin, C.T., Ng, H.K.T., 2003. Point and interval estimation for Gaussian distribution, based on progressively Type-II censored samples. IEEE Transactions on Reliability 52, 90 - 95.

[2] Balasooriya, U., Balakrishnan, N., 2000. Reliability sampling plans for log-normal distribution, based on progressively-censored samples. IEEE Transactions on Reliability 49, $199-203$.

[3] Banerjee, A., Kundu, D., 2008. Inference based on Type-II hybrid censored data from a Weibull distribution. IEEE Transactions on Reliability 57, 369-378. 
[4] Chen, S., Bhattacharya, G.K., 1988. Exact confidence bounds for an exponential parameter under hybrid censoring. Communications in Statistics - Theory and Methods 17, 18571870.

[5] Childs, A., Chandrasekhar, B., Balakrishnan, N., Kundu, D., 2003. Exact inference based on type-I and type-II hybrid censored samples from the exponential distribution. Annals of the Institute of Statistical Mathematics 55, 319-330.

[6] Dempster, A.P., Laird, N.M., Rubin, D.B., 1977. Maximum likelihood from incomplete data via the EM algorithm. Journal of the Royal Statistical Society, Ser. B 39, 1 - 38.

[7] Draper, N., Guttman, I., 1987. Bayesian analysis of hybrid life tests with exponential failure times. Annals of the Institute of Statistical Mathematics 39, 219 - 225.

[8] Ebrahimi, N., 1990. Estimating the parameter of an exponential distribution from hybrid life test. Journal of Statistical Planning and Inference 23, 255 - 261.

[9] Ebrahimi, N., 1992. Prediction intervals for future failures in exponential distribution under hybrid censoring. IEEE Transactions on Reliability 41, 127 - 132.

[10] Epstein, B., 1954. Truncated life tests in the exponential case. Annals of Mathematical Statistics 25, $555-564$.

[11] Fairbanks, K., Madson, R., Dykstra, R., 1982. A confidence interval for an exponential parameter from a hybrid life test. Journal of the American Statistical Association 77, $137-140$.

[12] Gupta, R.D., Kundu, D., 1998. Hybrid censoring schemes with exponential failure distribution. Communications in Statistics - Theory and Methods 27, 3065 - 3083. 
[13] Gupta, R.D., Kundu, D., 2006. On the comparison of Fisher information matrices of the Weibull and generalized exponential distributions, Journal of Statistical Planning and Inference 136, 3130 - 3144.

[14] Jeong, H.S., Park, J.I., Yum, B.J., 1996. Development of (r, T) hybrid sampling plans for exponential lifetime distributions. Journal of Applied Statistics 23, 601 - 607.

[15] Johnson, N.L., Kotz, S., Balakrishnan, N., 1995. Continuous Univariate Distribution, 2nd edition. Wiley, New York.

[16] Kundu, D., 2007. On hybrid censored Weibull distribution. Journal of Statistical Planning and Inference 137, 2127 - 2142.

[17] Kundu, D., 2008. Bayesian inference and life testing plan for Weibull distribution in presence of progressive censoring. Technometrics 50, 144 - 154 .

[18] Kundu, D., Pradhan, B. 2009. Estimating the parameters of the generalized exponential distribution in presence of hybrid censoring. Communications in Statistics - Theory and Methods 38, 2030 - 2041. .

[19] Lam, Y., 1994. Bayesian Variable Sampling Plans for the Exponential Distribution With Type I Censoring. The Annals of Statistics 22, 696 - 711.

[20] Lawless, J.F., 2003. Statistical Models and Methods for Lifetime Data. Wiley, New York.

[21] Lin, Y.P., Liang, T., Huang, W. T., 2002. Bayesian Sampling Plans for Exponential Distribution Based on Type I Censoring Data. Annals of the Institute of Statistical Mathematics 54, 100113.

[22] Louis, T.A., 1982. Finding the observed matrix when using the EM algorithm. Journal of the Royal Statistical Society, Ser. B 44, 226 - 233. 
[23] MIL-STD-781-C, 1977. Reliability Design Qualifications and Production Acceptance Test, Exponential Distribution. U.S. Government Printing Office, Washington, D.C..

[24] Ng, H.K.T., Chan, P.S., Balakrishnan, N., 2002. Estimation of parameters from progressively censored data using EM algorithm. Computational Statistics and Data Analysis $39,371-386$.

[25] Ng, H.K.T., Chan, P.S., Balakrishnan, N., 2004. Optimal Progressive Censoring Plans for the Weibull Distribution. Technometrics 46, 470481.

[26] Tiku, M.L., Tan, W.Y., Balakrishnan, N., 1986. Robust Inference, Marcel Dekker.

[27] Zhang, Y., Meeker, W.Q., 2005. Bayesian life test planning for the Weibull distribution with given shape parameter. Metrika 61, 237 - 249.

\section{APPENDIX:}

Here we derive the Fisher information matrix. The Fisher information matrix is given by

$$
I=\left[\begin{array}{cc}
-E\left(\frac{\partial^{2} L}{\partial \mu^{2}}\right) & -E\left(\frac{\partial^{2} L}{\partial \mu \partial \sigma}\right) \\
-E\left(\frac{\partial^{2} L}{\partial \sigma \partial \mu}\right) & -E\left(\frac{\partial^{2} L}{\partial \sigma^{2}}\right)
\end{array}\right]=\left[\begin{array}{ll}
I_{11} & I_{12} \\
I_{21} & I_{22}
\end{array}\right], \text { say }
$$

where

$$
\begin{gathered}
\frac{\partial^{2} L}{\partial \mu^{2}}=-\frac{r}{\sigma^{2}}+\frac{(n-r) \phi\left(z^{*}\right)}{\sigma^{2}\left(\Phi\left(-z^{*}\right)\right)}\left[z^{*}-\frac{\phi\left(z^{*}\right)}{\Phi\left(-z^{*}\right)}\right] \\
\frac{\partial^{2} L}{\partial \sigma^{2}}=\frac{r}{\sigma^{2}}-\frac{3}{\sigma^{4}} \sum_{i=1}^{r}\left(x_{i: n}-\mu\right)^{2}+\frac{(n-r) z^{*} \phi\left(z^{*}\right)}{\sigma^{2}\left(\Phi\left(-z^{*}\right)\right)}\left[\left(z^{*}\right)^{2}-2-\frac{z^{*} \phi\left(z^{*}\right)}{\Phi\left(-z^{*}\right)}\right], \\
\frac{\partial^{2} L}{\partial \mu \partial \sigma}=-\frac{2}{\sigma^{3}} \sum_{i=1}^{r}\left(x_{i: n}-\mu\right)+\frac{(n-r) \phi\left(z^{*}\right)}{\sigma^{2}\left(\Phi\left(-z^{*}\right)\right)}\left[\left(z^{*}\right)^{2}-1-\frac{z^{*} \phi\left(z^{*}\right)}{\Phi\left(-z^{*}\right)}\right], \\
I_{11}=\frac{E(r)}{\sigma^{2}}-\frac{n-E(r)}{\sigma^{2}} A,
\end{gathered}
$$




$$
\begin{aligned}
& I_{22}=-\frac{E(r)}{\sigma^{2}}+\frac{3}{\sigma^{4}} E(r) E(\log Y-\mu)^{2}-[n-E(r)] B=2 \frac{E(r)}{\sigma^{2}}-[n-E(r)] B, \\
& I_{12}=\frac{2}{\sigma} E(r) E(\log Y-\mu)-[n-E(r)] C=-[n-E(r)] C, \\
& E(r)=R \sum_{i=R}^{n}\left(\begin{array}{c}
n \\
i
\end{array}\right)[\Phi(V)]^{i}[1-\Phi(V)]^{n-i}+\sum_{i=0}^{R-1} i\left(\begin{array}{c}
n \\
i
\end{array}\right)[\Phi(V)]^{i}[1-\Phi(V)]^{n-i}, \\
& A=\frac{\phi(V)}{1-\Phi(V)}\left[V-\frac{\phi(V)}{1-\Phi(V)}\right] \times P\left(y_{R: n}>T\right) \\
& +E\left[\frac{\phi\left(Z_{R}\right)}{1-\Phi\left(Z_{R}\right)}\left\{Z_{R}-\frac{\phi\left(Z_{R}\right)}{1-\Phi\left(Z_{R}\right)}\right\} \mid y_{R: n} \leq T\right] \times P\left(y_{R: n} \leq T\right) \\
& =\frac{\phi(V)}{1-\Phi(V)}\left[V-\frac{\phi(V)}{1-\Phi(V)}\right] P\left(y_{R: n}>T\right) \\
& +R\left(\begin{array}{c}
n \\
R
\end{array}\right) \int_{0}^{\Phi\left(\frac{\ln T-\mu}{\sigma}\right)} \phi\left(\Phi^{-1}(y)\right)\left\{\Phi^{-1}(y)-\frac{\phi\left(\Phi^{-1}(y)\right)}{1-y}\right\} y^{R-1}(1-y)^{n-R} d y, \\
& B=\frac{V \phi(V)}{1-\Phi(V)}\left[V^{2}-2-\frac{V \phi(V)}{1-\Phi(V)}\right] \times P\left(y_{R: n}>T\right) \\
& +E\left[\frac{Z_{R} \phi\left(Z_{R}\right)}{1-\Phi\left(Z_{R}\right)}\left\{Z_{R}^{2}-2-\frac{Z_{R} \phi\left(Z_{R}\right)}{1-\Phi\left(Z_{R}\right)}\right\} \mid y_{R: n} \leq T\right] \times P\left(y_{R: n} \leq T\right) \\
& =\frac{V \phi(V)}{1-\Phi(V)}\left[V^{2}-2-\frac{V \phi(V)}{1-\Phi(V)}\right] \times P\left(y_{R: n}>T\right) \\
& +R\left(\begin{array}{c}
n \\
R
\end{array}\right) \int_{0}^{\Phi\left(\frac{\ln T-\mu}{\sigma}\right)} \Phi^{-1}(y) \phi\left(\Phi^{-1}(y)\right)\left\{\left(\Phi^{-1}(y)\right)^{2}-2\right. \\
& \left.-\frac{\Phi^{-1}(y) \phi\left(\Phi^{-1}(y)\right)}{1-y}\right\} y^{R-1}(1-y)^{n-R} d y \\
& C=\frac{\phi(V)}{1-\Phi(V)}\left[V^{2}-1-\frac{V \phi(V)}{1-\Phi(V)}\right] \times P\left(y_{R: n}>T\right) \\
& +E\left[\frac{\phi\left(Z_{R}\right)}{1-\Phi\left(Z_{R}\right)}\left\{Z_{R}^{2}-1-\frac{Z_{R} \phi\left(Z_{R}\right)}{1-\Phi\left(Z_{R}\right)}\right\} \mid y_{R: n} \leq T\right] \times P\left(y_{R: n} \leq T\right) \\
& =\frac{\phi(V)}{1-\Phi(V)}\left[V^{2}-1-\frac{V \phi(V)}{1-\Phi(V)}\right] \times P\left(y_{R: n}>T\right) \\
& +R\left(\begin{array}{c}
n \\
R
\end{array}\right) \int_{0}^{\Phi\left(\frac{\ln T-\mu}{\sigma}\right)} \phi\left(\Phi^{-1}(y)\right)\left\{\left(\Phi^{-1}(y)\right)^{2}-1\right. \\
& \left.-\frac{\Phi^{-1}(y) \phi\left(\Phi^{-1}(y)\right)}{1-y}\right\} y^{R-1}(1-y)^{n-R} d y,
\end{aligned}
$$




$$
\text { and } \begin{aligned}
P\left(y_{R: n}>T\right)= & R\left(\begin{array}{c}
n \\
R
\end{array}\right) \int_{T}^{\infty}[F(x)]^{R-1}[1-F(x)]^{n-R} f(x) d x \\
& =R\left(\begin{array}{c}
n \\
R
\end{array}\right) \int_{\Phi(V)}^{1} z^{R-1}(1-z)^{n-R} d z
\end{aligned}
$$


Table 1: Average estimate, the corresponding MSE in parentheses and 95\% coverage percentage for $n=25, T=55$

\begin{tabular}{|c|c|c|c|}
\hline & $\mathrm{R}=15$ & $\mathrm{R}=20$ & $\mathrm{R}=25$ \\
\hline AMLE & $4.00155(0.00158), 93.5$ & $4.00181(0.00141), 95.2$ & $4.00154(0.00144), 95.2$ \\
& $0.14551(0.00120), 88.6$ & $0.14629(0.00114), 89.8$ & $0.14614(0.00116), 90.0$ \\
\hline \multirow{2}{*}{ MLE } & $4.00190(0.00156), 93.8$ & $4.00209(0.00139), 95.5$ & $4.00184(0.00142), 95.5$ \\
& $0.14578(0.00119), 88.9$ & $0.14653(0.00113), 90.1$ & $0.14639(0.00115), 90.3$ \\
\hline
\end{tabular}

Table 2: Average estimate, the corresponding MSE in parentheses and 95\% coverage percentage for $n=40, T=55$

\begin{tabular}{|c|c|c|c|}
\hline & $\mathrm{R}=30$ & $\mathrm{R}=35$ & $\mathrm{R}=40$ \\
\hline AMLE & $4.00076(0.00077), 95.1$ & $4.00096(0.00086), 95.0$ & $4.00096(0.00086), 95.0$ \\
& $0.14802(0.00062), 92.2$ & $0.14797(0.00069), 92.0$ & $0.14797(0.00069), 92.0$ \\
\hline MLE & $4.00085(0.00077), 95.2$ & $4.00111(0.00086), 95.1$ & $4.00111(0.00086), 95.1$ \\
& $0.14812(0.00061), 92.4$ & $0.14812(0.00068), 92.3$ & $0.14812(0.00068), 92.3$ \\
\hline
\end{tabular}

Table 3: Average estimate, the corresponding MSE in parentheses and 95\% coverage percentage for $n=25, T=65$

\begin{tabular}{|c|c|c|c|}
\hline & $\mathrm{R}=15$ & $\mathrm{R}=20$ & $\mathrm{R}=25$ \\
\hline AMLE & $3.99638(0.00113), 91.6$ & $3.99905(0.00097), 92.9$ & $4.00067(0.00093), 93.9$ \\
& $0.14065(0.00097), 86.9$ & $0.14384(0.00069), 89.2$ & $0.14633(0.00058), 90.7$ \\
\hline \multirow{2}{*}{ MLE } & $3.99668(0.00091), 91.8$ & $3.99926(0.00096), 93.1$ & $4.00091(0.00092), 94.1$ \\
& $0.14090(0.00096), 87.1$ & $0.14413(0.00067), 89.8$ & $0.14675(0.00057), 91.6$ \\
\hline
\end{tabular}


Table 4: Average estimate, the corresponding MSE in parentheses and $95 \%$ coverage percentage for $n=40, T=65$.

\begin{tabular}{|c|c|c|c|}
\hline & $\mathrm{R}=30$ & $\mathrm{R}=35$ & $\mathrm{R}=40$ \\
\hline AMLE & $3.99919(0.00062), 93.4$ & $4.00016(0.00060), 93.5$ & $4.00068(0.00059), 93.8$ \\
& $0.14586(0.00044), 91.5$ & $0.14695(0.00037), 92.1$ & $0.14769(0.00035), 92.6$ \\
\hline MLE & $3.99933(0.00062), 93.6$ & $4.00027(0.00060), 93.6$ & $4.00077(0.00059), 93.9$ \\
& $0.14603(0.00043), 91.8$ & $0.14711(0.00037), 92.5$ & $0.14783(0.00035), 92.9$ \\
\hline
\end{tabular}

Table 5: Information measure $I\left(n, R_{n}, T_{n}\right)$ for $\left(n, R_{n}, T_{n}\right), 1 \leq n \leq n_{1}$.

\begin{tabular}{|c|c|c|c|c|c|c|c|c|c|}
\hline$n$ & \multirow{2}{*}{$T_{n}$} & \multicolumn{7}{|c|}{$R_{n}$} \\
\cline { 3 - 10 } & & 1 & 2 & 3 & 4 & 5 & 6 & 7 & 8 \\
\hline 1 & 8.8 & 0.0383 & & & & & & & \\
\hline 2 & 7.6 & 0.0439 & 0.0767 & & & & & & \\
\hline 3 & 6.4 & 0.0435 & 0.0879 & 0.1158 & & & & & \\
\hline 4 & 5.2 & 0.0419 & 0.0902 & 0.1315 & 0.1573 & & & & \\
\hline 5 & 4.0 & 0.0402 & 0.0883 & 0.1373 & 0.1774 & 0.2061 & & & \\
\hline 6 & 2.8 & 0.0389 & 0.0848 & 0.1385 & 0.1880 & 0.2314 & 0.2679 & & \\
\hline 7 & 1.6 & 0.0379 & 0.0813 & 0.1368 & 0.1926 & 0.2390 & 0.2717 & 0.2890 & \\
\hline 8 & 0.4 & 0.0244 & 0.0295 & 0.0296 & 0.0296 & 0.0296 & 0.0296 & 0.0296 & 0.0296 \\
\hline
\end{tabular}




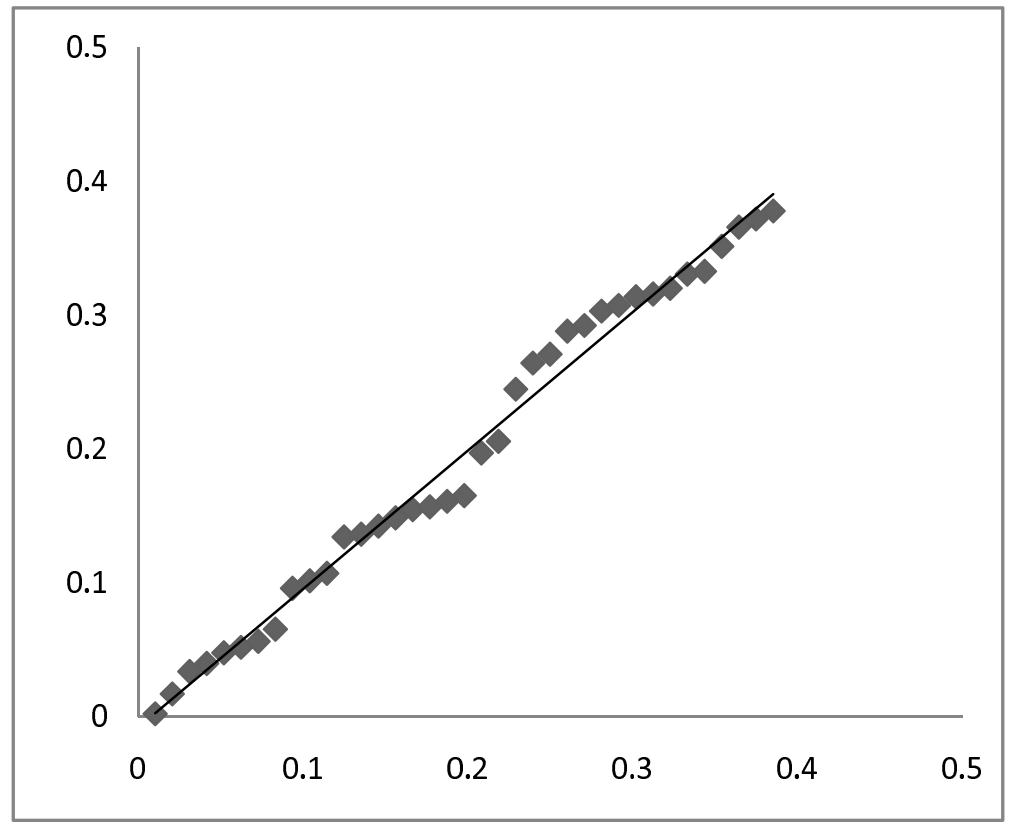

Figure 1: PP Plot 